\title{
TURBULENCE IN THE SUB-ALFVÉNIC SOLAR WIND DRIVEN BY REFLECTION OF LOW-FREQUENCY ALFVÉN WAVES
}

\author{
A. Verdini ${ }^{1}$, M. Velli ${ }^{2,3}$, and E. Buchlin ${ }^{4}$ \\ ${ }^{1}$ Observatoire Royale de Belgique, 3 Avenue Circulaire, 1180, Bruxelles, Belgium; e-mail: verdini@ oma.be \\ 2 Dip. di Astronomia e Scienza dello Spazio, Univ. di Firenze, Largo E. Fermi 3, 50125 Firenze, Italy \\ ${ }^{3}$ Jet Propulsion Laboratory, California Institute of Technology, 4800 Oak Grove Drive, Pasadena, CA 91109, USA \\ ${ }^{4}$ Institut d'Astrophysique Spatiale, CNRS - Université Paris Sud, Bât. 121, 91405 Orsay Cedex, France \\ Received 2009 March 17; accepted 2009 May 15; published 2009 July 2
}

\begin{abstract}
We study the formation and evolution of a turbulent spectrum of Alfvén waves driven by reflection off the solar wind density gradients, starting from the coronal base up to 17 solar radii, well beyond the Alfvénic critical point. The background solar wind is assigned and two-dimensional shell models are used to describe nonlinear interactions. We find that the turbulent spectra are influenced by the nature of the reflected waves. Close to the base, these give rise to a flatter and steeper spectrum for the outgoing and reflected waves, respectively. At higher heliocentric distance both spectra evolve toward an asymptotic Kolmogorov spectrum. The turbulent dissipation is found to account for at least half of the heating required to sustain the background imposed solar wind and its shape is found to be determined by the reflection-determined turbulent heating below 1.5 solar radii. Therefore, reflection and reflection-driven turbulence are shown to play a key role in the acceleration of the fast solar wind and origin of the turbulent spectrum found at 0.3 AU in the heliosphere.
\end{abstract}

Key words: MHD - solar wind - turbulence - waves

\section{INTRODUCTION}

Recent high-resolution observations from Hinode (De Pontieu et al. 2007) and the coronal multi-channel polarimeter (CoMP) (Tomczyk et al. 2007) have shown that the solar atmosphere is pervaded by Alfvénic (or kink-like, e.g., Van Doorsselaere et al. 2008) oscillations: observed in jets, spicules, or in coronal loops, velocity and magnetic field oscillations $(\delta b, \delta u)$ are coupled and propagate at speeds close to the Alfvén speed. Far from the Sun, between $0.3 \mathrm{AU}$ and several AU, in situ data show that in the frequency range $10^{-4} \mathrm{~Hz} \lesssim f \lesssim 10^{-2} \mathrm{~Hz}$, fluctuations in magnetic field and velocity $\delta b$ and $\delta u$ possess many of the properties of outward-propagating "spherically polarized" Alfvén waves, namely, quasi-incompressibility, correlated oscillations, and a constant (total) magnetic field intensity, while at the same time revealing their turbulent nature through a welldeveloped power-law frequency spectrum, with a break separating different power law slopes of -1 and -1.6 which moves to lower frequencies with increasing distance from the Sun (Bavassano et al. 1982; Tu et al. 1984). The $\delta u \cdot \delta b$ correlation, upon which the propagation direction determination is made, depends on the frequency considered and varies with distance (Bavassano et al. 2000a, 2000b) and latitude (Grappin 2002), typically in the range $1 / 2<\left|(\delta u \cdot \delta b / \sqrt{4 \pi \rho}) /\left(\delta u^{2}+\delta b^{2} / 4 \pi \rho\right)\right|<1$ (perfect correlation corresponding to 1$)$. These facts suggest that the inward traveling wave-mode component, required for nonlinear couplings between incompressible fluctuations, must indeed be present.

This component might be generated locally between $0.3 \mathrm{AU}$ and $1 \mathrm{AU}$ by shear, compressible or pick-up ion interactions, or it could be already formed in the sub-Alfvénic corona and, later on, nonlinearly advected into the heliosphere by the solar wind, the hypothesis we consider here.

The dynamics inside the Alfvénic point region is of primary importance to understand the origin of the spectrum one finds at $0.3 \mathrm{AU}$ and to determine whether it has any role in accelerating the solar wind. The variation of the propagation speed induced by density gradients in the stratified corona and accelerating so- lar wind causes outward Alfvén waves to be reflected, predominantly at lower frequencies, hence triggering the incompressible cascade. The power dissipated by the cascade contributes to coronal heating, also modifying the overall turbulent pressure gradient, fundamental to the acceleration of the fast solar wind.

While there are several studies on the linear propagation and reflection of Alfvén waves in the sub-Alfvénic corona and solar wind (Hollweg 1978; Heinemann \& Olbert 1980; Velli 1993; Hollweg \& Isenberg 2007), excepting phenomenological models with an essentially dimensional estimate of the role of turbulent heating (Hollweg et al. 1982; Dmitruk et al. 2001; Cranmer \& van Ballegooijen 2005; Verdini \& Velli 2007) very few of them have considered nonlinear interactions. Velli et al. $(1989,1990)$ studied the turbulent cascade sustained by reflected waves in the super-Alfvénic solar wind, while Dmitruk et al. (2002) considered the same mechanism in the sub-Alfvénic corona below $3 R_{\odot}$, hence neglecting the solar wind.

In this Letter, we will extend these studies following the development of the turbulent cascade from the base of the corona up to $17 R_{\odot}$, well beyond the Alfvénic critical point (located at about $13 R_{\odot}$ in the solar wind model adopted). Direct numerical simulations are still prohibitively costly in terms of computational time, so nonlinear interactions are simulated using a two-dimensional shell model (Buchlin \& Velli 2007) which simplifies nonlinear interactions but still allows four decades in the perpendicular wavenumber space to be covered while rigorously treating the propagation and reflection of waves along the radial mean magnetic field.

\section{MODEL DESCRIPTION}

The equations describing the propagation of Alfvén waves in an inhomogeneous medium are derived from magnetohydrodynamics (MHD), assuming that the large-scale fields are stationary and separating the time-fluctuating fields from the large-scale averages (Heinemann \& Olbert 1980; Velli 1993). Therefore, the large-scale magnetic field, bulk wind flow, and density ( $B, U, \rho$, respectively) appear as specified coefficients in the MHD equations for the fluctuations. 


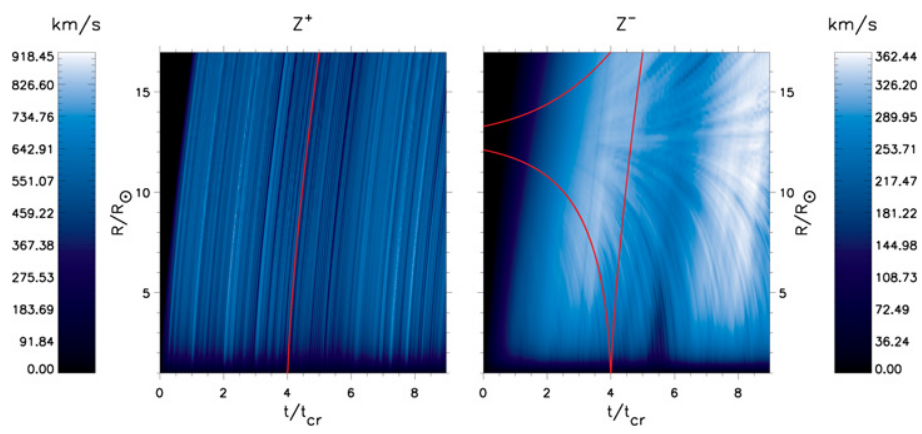

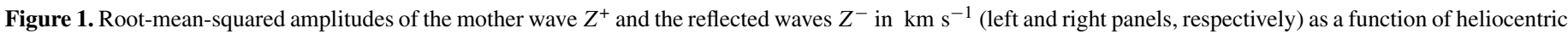

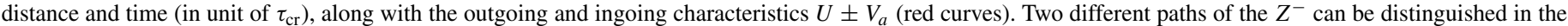

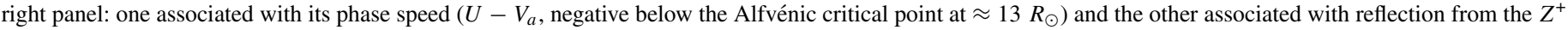
which forces the fluctuations to follow the outgoing wave (with phase speed $U+V_{a}$ ).

We consider a magnetic flux tube centered in a polar coronal hole, which expands super-radially with a (nondimensional) area $A(r)=r^{2} f(r)$ first prescribed by Kopp \& Holzer (1976) and Munro \& Jackson (1977). Distances are normalized to the solar radius, and the coefficients are given by $r_{0}=1.31, \sigma=0.5, f_{\max }=7.26$ (respectively, the location, width, and asymptotic value of the super-radial expansion) so that $A(1)=1$. The field becomes $B(r)=B_{\odot} / A(r)$ where we take $B_{\odot}=10 \mathrm{G}$. The wind speed and density $U(r), \rho(r)$ are obtained solving the one-dimensional momentum equation with an assigned temperature $T(r), T_{\odot}=410^{5} \mathrm{~K}$ and a numerical density at the coronal base $n_{\odot}=510^{7} \mathrm{~cm}^{-3}$ (see Verdini \& Velli 2007 and references therein for details on the equation and on the temperature profile). The resulting wind is supersonic far from the Sun with $U_{1 \mathrm{AU}} \approx 750 \mathrm{~km} \mathrm{~s}^{-1}$ and $n_{1 \mathrm{AU}} \approx 3 \mathrm{~cm}^{-3}$. The Alfvén critical point $\left(r_{a}\right)$ is at about $13 R_{\odot}$, the sonic critical point is at about $1.9 R_{\odot}$, the Alfvén speed $V_{a}=B / \sqrt{4 \pi \rho}$ at the base is $V_{a, \odot} \approx 1000 \mathrm{~km} \mathrm{~s}^{-1}$ and has a maximum $V_{a, \max } \approx 3500 \mathrm{~km} \mathrm{~s}^{-1}$ at $r=1.5 R_{\odot}$. At the end of the domain $\left(17 R_{\odot}\right) U \approx 740 \mathrm{~km} \mathrm{~s}^{-1}$ and $V_{a} \approx 630 \mathrm{~km} \mathrm{~s}^{-1}$.

We assume $\delta u$ to be incompressible and transverse with respect to $B$. The momentum and induction equations for $\delta u$ and $\delta b$ are written in terms of the Elsässer fields $Z^{ \pm}=\delta u \mp \delta b / \sqrt{4 \pi \rho}$, corresponding to Alfvén waves which propagate respectively outward and inward in the solar wind reference frame.

Substituting the nonlinear terms, which act in planes perpendicular to the radial direction, with a two-dimensional MHD shell model representation (in the form given by Biskamp 1994) and assuming radial propagation finally yields the model equations for $Z^{ \pm}=Z^{ \pm}\left(r, k_{\perp}\right)=Z_{n}^{ \pm}(r)$ :

$$
\begin{aligned}
& \frac{\partial Z_{n}^{ \pm}}{\partial t}+\left(U \pm V_{a}\right) \frac{\partial Z_{n}^{ \pm}}{\partial r}+\frac{1}{2}\left(U \mp V_{a}\right)\left(\frac{d \log V_{a}}{d r}+\frac{d \log A}{d r}\right) Z_{n}^{ \pm} \\
& -\frac{1}{2}\left(U \mp V_{a}\right)\left(\frac{d \log V_{a}}{d r}\right) Z_{n}^{\mp}=-k_{n}^{2}\left(v^{+} Z_{n}^{ \pm}+v^{-} Z_{n}^{\mp}\right) \\
& +\mathrm{ik}_{\mathrm{n}}\left(\mathrm{T}_{\mathrm{n}}^{ \pm}\right)^{*} .
\end{aligned}
$$

The complex scalar values, $u_{n}=\left(Z_{n}^{+}+Z_{n}^{-}\right) / 2, b_{n}=\left(Z_{n}^{-}-\right.$ $\left.Z_{n}^{+}\right) / 2$, represent the velocity and magnetic (in velocity units) field fluctuations corresponding to the scale $\lambda_{n}=\lambda_{0} 2^{-n}=$ $2 \pi / k_{n}, n$ is the shell index, and $T_{n}^{ \pm}$accounts for nonlinear interactions of the form $Z_{l}^{+} Z_{m}^{-}$, with $l, m=n \pm 1, n \pm 2$. Finally, $v^{ \pm}=(\nu \pm \eta) / 2$ are combinations of the kinematic viscosity and the magnetic resistivity (we take $\eta=v$ ).

Simulations are carried out with the code Shell-Atm ${ }^{5}$ (Buchlin \& Velli 2007). The advection terms in Equation (1)

\footnotetext{
5 Modified to include the wind spherical expansion.
}

are computed with a second-order upwind scheme (Fromm scheme) which allows a good conservation of the phase of the fluctuation. Time is advanced with a third-order Runge-Kutta for the nonlinear part of the equations. The radial domain is decomposed in $\sim 25,000$ planes over a nonuniform grid while 21 shells are used for the nonlinear interactions. Transparent boundary conditions are imposed at the top for both waves, and at the bottom for the $Z^{-}$. Here, all the gradients are artificially set to zero, in order to avoid reflections. Energy is injected in the domain imposing the amplitude $Z_{n, \odot}^{+}=f_{n}(t)$ at the first three shells corresponding to length scale of the order of 8.000 $34.000 \mathrm{~km}\left(\lambda_{0, \odot}=0.02 R_{\odot}\right.$ in the shell model $)$, with $f(t)$ a function with a time correlation and periodicity $\tau^{*} \approx 1000 \mathrm{~s}$. The form of the function is given in Buchlin \& Velli (2007); here it is important to note that despite the correlation time is only $1000 \mathrm{~s}$, some low-frequency fluctuations are injected for long time series (as in the present simulation). Simulations last about 20 crossing timescales, $\tau_{\mathrm{cr}}=\int_{R_{\odot}}^{17 R_{\odot}} \approx 7000 \mathrm{~s}$, and timeaveraged quantities are computed on the last $10 \tau_{\mathrm{cr}}$, during which the system has an approximate stationary state.

\section{REFLECTION AND NONLINEARITIES}

Given that we start with an initial flux of $Z^{+}$at the coronal base, reflection is the only trigger for nonlinear interactions. In Figure 1, the root-mean-squared (rms) amplitudes $\left|Z^{ \pm}\right|=$ $\sqrt{\sum_{n}\left|Z_{n}^{ \pm}\right|^{2}}$ are plotted as a function of time and heliocentric distance. Two components of the reflected waves are clearly visible in the $Z^{-}$contours: a "classical" component $Z_{\text {class }}^{-}$, which propagate with the expected $U-V_{a}$ phase speed (negative below the Alfvénic critical point, $r_{a} \approx 13 R_{\odot}$ ) and an "anomalous" component $Z_{\text {anom }}^{-}$, which travels with the same speed as the mother wave, $U+V_{a}$. As shown analytically and numerically (Velli et al. 1990; Hollweg \& Isenberg 2007) this "anomalous" component is the direct product of reflection. Generally, in the presence of density gradients, for small values of the ratio $\epsilon=\alpha / \omega$, typical of the upper corona, with $\alpha=\tau_{R}^{-1}=\left|\left(U \mp V_{a}\right) V_{a}^{\prime} / V_{a}\right|$ in Equation (1), each field can be decomposed in a primary and secondary component. A $Z^{+}$ primary component is injected at the base, while the $Z^{-}$is made up of only the secondary component, given by reflection. In each plane, as the $Z^{+}$arrives, the secondary component can be seen as the result of a forcing term given by $\approx \alpha Z^{+}$, hence producing a wave which travels with the same phase speed and of the above amplitude. The value $Z^{-}=\epsilon Z^{+}$follows naturally by finding the "forced" solution to the linearized equation for $Z^{-}$. At a later time, as the $Z^{+}$has propagated away, the forcing 


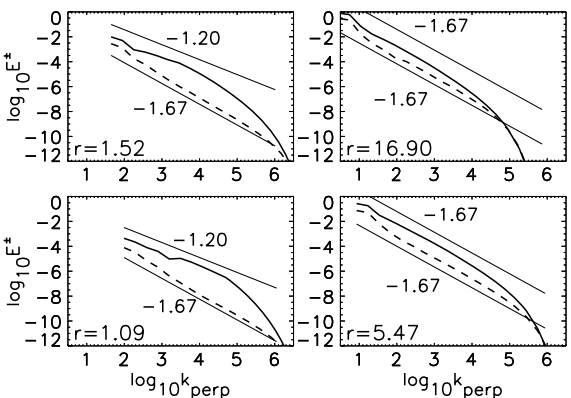

Figure 2. Energy spectra $E_{k}^{ \pm}$(solid and dashed lines, respectively) as a function of the perpendicular wavenumber in four different planes, as indicated at the bottom left of each panel $\left(r=R / R_{\odot}\right)$. Spectra are averaged in time and normalized to $\approx 10^{25} \mathrm{~cm}^{3} \mathrm{~s}^{-2}$, wavenumbers to $R_{\odot}^{-1}$. The thin lines are power law with the indicated slopes, plotted for reference.

disappears and the secondary component propagates backward with the classical phase speed. When a $Z^{+}$pulse wave is excited in the corona, $Z^{-}$appears as a halo spreading backward from the mother $Z^{+}$wave. Nonlinear interactions modifies the above picture, acting as a local (in a given plane) source for the $Z_{n}^{-}$ which is uncorrelated with respect to that given by reflection and hence generating waves propagating with classical phase speed $U-V_{a}$, i.e., a primary component.

Inward propagating waves have a very long propagation time at $r_{a}$ that slows down the overall relaxation toward a steady state: for example, in Figure 1 the increase of the $Z^{-}$amplitude at $t \approx 8 \tau_{\text {cr }}$ results from the superposition of the $Z_{\text {anom }}^{-}$with the backward propagating $Z_{\text {class }}^{-}$produced at $t \approx 3 \tau_{\mathrm{cr}}$.

The different nature of the reflected waves influences the spectral energy transfer. Generally speaking, while the wave amplitudes increase with distance the nonlinear timescales $\tau_{\mathrm{nl}}^{ \pm}=\left(k_{n} Z_{n}^{\mp}\right)^{-1}$ decrease, following the reflection coefficient and the flux tube expansion. At small wavenumbers $\tau_{\mathrm{nl}}^{-}<\tau_{\mathrm{nl}}^{+}$, the energy transfer is more efficient for the reflected waves, although the $Z^{+}$contribute more to the total energy dissipation. As a matter of fact, the $Z^{-}$develop an inertial range in the whole domain and the resulting cascade is more "aged." Their dynamics is governed by the nonlinear interactions which have shorter timescales (as $Z^{+}>Z^{-}$) and act for longer periods, the $Z^{-}$having a smaller propagation speed.

The contribution of coherent interactions $Z^{+} Z_{\text {anom }}^{-}$in shaping the energy spectra $E_{k}^{ \pm}=\left|Z_{n}^{ \pm}\right|^{2} /\left(4 k_{n}\right)$ can be seen in the low corona, before $1.2 R_{\odot}$ (left panels of Figure 2 ). In fact at $1.09 R_{\odot}$, assuming waves of period $T=1000 \mathrm{~s}, \epsilon \approx 0.07$ $\left(\epsilon_{\max }=0.16\right.$ at the coronal base), hence in the presence of lower frequency fluctuations (injected by the forcing) reflection is relatively high. At about $1.5 R_{\odot}, V_{a}$ has a maximum and reflection vanishes, so that the $Z^{-}$are not produced locally but rather propagate from above. This inhibits the spectral energy flux of $Z^{+}$that becomes negative at large scales. The inverse spectral transfer is responsible of the flatter part of the $E^{+}$spectrum one finds at small $k_{\perp}$. Further out reflection is negligible and the spectra are the remnant of those produced in the inner layers, showing a slow evolution toward the asymptotic state in which $E^{+} \gtrsim 3 E^{-}$. The spectral energy flux is given by the interactions $Z^{+} Z_{\text {class }}^{-}$that are subject to the "Alfvén effect" (a longer cascade timescale due to the nonlinear interactions between wavepackets propagating in opposite directions), producing the same spectral slopes $-5 / 3$ for $E^{ \pm}$(right panels of Figure 2).

Note that the largest perpendicular scale is proportional to the flux tube width, $\lambda_{0}(r)=\lambda_{0, \odot} \sqrt{A(r)}$, hence the same number of shells spans a $k_{\perp}$ interval that shifts to smaller values with increasing $r$.

The asymptotic slope $-5 / 3$ and that of the reflectiondominated spectra can be deduced from the expression of spectral energy flux of the shell model $\left(a_{1}=11 / 24, a_{2}=\right.$ $\left.1-a_{1}, a_{3}=-15 / 24\right)$,

$$
\begin{aligned}
\Pi_{n}^{ \pm}= & -\operatorname{Im}\left[k_{n} Z_{n}^{+}\left(a_{1} Z_{n+1}^{+} Z_{n+2}^{-}+a_{2} Z_{n+1}^{-} Z_{n+2}^{+}\right)\right. \\
& \left.+k_{n-1} Z_{n+1}^{+}\left(a_{2} Z_{n-1}^{+} Z_{n}^{-}+a_{3} Z_{n-1}^{-} Z_{n}^{+}\right)\right] .
\end{aligned}
$$

When reflection is negligible one can assume that the $Z^{+}$ and $Z^{-}$are uncorrelated $\left\langle Z^{+} Z^{-*}\right\rangle_{t} \approx 0$. Assuming a power law for the spectral energies, $E_{n}^{ \pm} \propto\left(k_{n} / k_{0}\right)^{p_{ \pm}}$, substituting $Z_{n}^{ \pm}=2\left(k_{n} E_{n}^{ \pm}\right)^{1 / 2}$ in Equation (2) one finds that $\Pi_{n}^{ \pm}$is independent of the shell index $n$ when $p^{+}=p^{-}=-5 / 3$. For a given plane, it implies a constant normalized cross helicity $\sigma_{c}=\left(E^{+}-E^{-}\right) /\left(E^{+}+E^{-}\right)$in the inertial range, with a cross helicity spectrum $H_{c}=E^{+}-E^{-}$of the same slope as $E^{+}$, in contrast to EDQNM closure models, which, including nonlocal interactions in Fourier space, predict a distinct steeper spectrum, $H_{c} \propto k^{-2}$ (Grappin et al. 1983). From the evolution of the spectra (right panels) one can see that the normalized cross helicity decreases with $r$ because of two factors, the general increase of the total energy and the decrease of $H_{c}$ at all scales. The former is due to the approximate conservation of the total wave action density. The latter results from the competition of the linear coupling (reflection), which forces $Z^{-} \lesssim Z^{+}$for low-frequency fluctuations, with the nonlinear coupling, which damps the nondominant wave population, the $Z^{-}$(Dmitruk et al. 2001). Indeed the $E^{ \pm}$spectra look the same at small wavenumbers (corresponding to $n=0,1,2$ ) in which lowfrequency fluctuations reside.

When reflection is high the two fields are strongly coupled and one can assume that the growth of $Z_{n}^{-}$has contributions both from the nonlinear cascade and direct generation through reflection $\approx \epsilon_{n} Z_{n}^{+}$. The reflected contribution shares the phase properties of $Z_{n}^{+}$so interactions due to reflection are coherent below $1.2 R_{\odot}$ and the cascade may be isotropic for small wavenumbers in the $k_{\perp}-k_{\|}$plane. For the first seven shells (2 orders of magnitude), one would find $\omega_{n}=k_{n} V_{a}, \epsilon_{n} \propto \lambda^{-n}$ and the following scaling for the spectra $E^{+} \propto k^{-1}, E^{-} \propto k^{-3}$ (Velli et al. 1990). If the nonlinear cascade is somehow inhibited, keeping fixed the total energy in the parallel wavenumbers, low frequencies are confined to smaller $n$ and $\epsilon_{n} \propto \lambda^{-n p}$ with $p<1$, yielding a steeper (flatter) spectrum for $E^{+}\left(E^{-}\right)$compared to the isotropic case. In the present simulations, $\epsilon_{1.09} R_{\odot} \propto \lambda^{0.7}$ for $n<8$ which yields $p^{+}=1.2, p^{-}=2.6$ as in the bottom left panel of Figure 2.

The resulting turbulent heating is computed from Equation (1) multiplied by $\left(Z_{n}^{ \pm}\right)^{*}$ and summed over the shell index. The total energy dissipation per unit mass $H=Q / \rho=$ $1 / 2 v \sum_{n} k_{n}^{2}\left(\left|Z_{n}^{+}\right|^{2}+\left|Z_{n}^{-}\right|^{2}\right)$ increases in the low corona and decreases exponentially in the sub-Alfvénic region of the wind, as expected. For $\delta u_{\odot} \approx 49 \mathrm{~km} \mathrm{~s}^{-1}$ (solid line in Figure 3), it is also very close to the heating required to sustain the specified background wind (red line in Figure 3): the location and height of the peak coincide, although more energy is dissipated in the upper corona. This would produce a faster wind, not altering too much the mass flux, since the peak of the dissipation is close to the sonic critical point (Hansteen et al. 1999).

For smaller values of $\delta u_{\odot}$ a better agreement is found in the decreasing part, the rapid increase in the low corona is still reproduced but the peak intensity is not attained. Despite the 


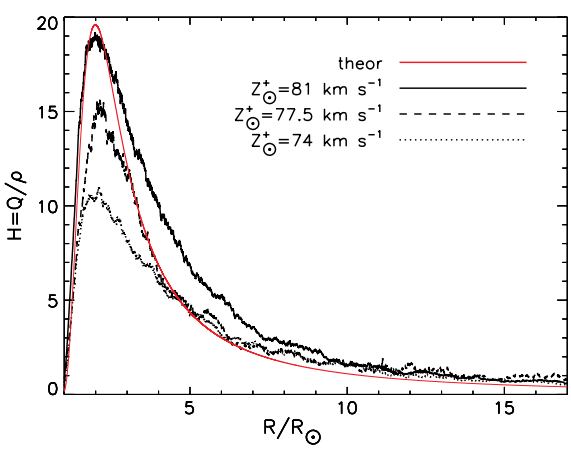

Figure 3. Time-averaged heating per unit mass as a function of distance for different root-mean-squared amplitudes at the coronal base $Z_{\odot}^{+}$. Also plotted in red is the heating function necessary to sustain the background specified solar wind. $H$ is in units of $\approx 3 \times 10^{10} \mathrm{~cm}^{2} \mathrm{~s}^{-3}$.

fact that the spectra and the spectral fluxes possess the same properties, for $\delta u_{\odot} \approx 45$ and $42 \mathrm{~km} \mathrm{~s}^{-1}$ the peak intensity decreases by a factor $1 / 4$ and $1 / 2$, respectively (dashed and dotted line in Figure 3). It turns out that the amount of dissipation is very sensitive to the level of velocity fluctuations at the base of the corona. In fact, $\delta u$ is determined by the injection of $Z^{+}$at the coronal base but also by the response of the atmosphere and by the nonlinear interactions (the level of $Z^{-}$). In contrast the peak dissipation seems to scale linearly with the rms amplitude $Z_{\odot}^{+}$although further studies (dependence on the frequency, on the nonlinear interactions in the shell model, on the imposed wind) are necessary to define the scaling precisely.

\section{DISCUSSION}

We have studied the propagation, reflection, and nonlinear interaction of Alfvén waves from the base of the corona up to 17 solar radii, well beyond the Alfvénic critical point. For the first time two-dimensional shell models have been applied to account for nonlinear interactions in magnetically open regions on the Sun, such as coronal holes. Thanks to such a simplification, compared to MHD or reduced MHD direct numerical simulations, it is possible to follow the development of a turbulent spectrum in the expanding solar wind, where waves are continuously reflected by the gradients in mean fields. Reflected waves are made of two components, one propagating with the characteristic phase speed $U-V_{a}\left(Z_{\text {class }}^{-}\right)$ and the other following the path of the outgoing wave with speed $U+V_{a}\left(Z_{\text {anom }}^{-}\right)$, a confirmation of previous linear results (Velli et al. 1989; Hollweg \& Isenberg 2007) which hold in a similar way also in the nonlinear regime. For typical coronal parameters, we find $Z^{-}=\epsilon(r, \omega) Z^{+} \propto V_{a}^{\prime} Z^{+}$, in contrast to $Z^{-} \propto \lambda_{0} V_{a}^{\prime}$ (independent of $Z^{+}$) found in a strong turbulence regime (Dmitruk et al. 2002) in which $Z^{-}, \lambda_{0} \rightarrow 0$. Differences arise because the above limits impose a timescale ordering $\tau_{\mathrm{nl}}^{-}<<\tau_{\mathrm{nl}}^{+} \lesssim \tau_{R}<\tau_{\mathrm{cr}}$ which is not satisfied in our simulation, basically because $Z^{-} \nrightarrow 0$ even in case in which the outer scale of turbulence does not follow the flux tube expansion (similar to the limit $\lambda_{0} \rightarrow 0$ ).

Close to the coronal base one can distinguish the contribution to the spectral slope of the coherent nonlinear interactions $Z_{\text {anom }}^{-} Z^{+}$, which give $E^{+} \propto k^{-1.2}$, and the one from the incoherent nonlinear interaction $Z_{\text {class }}^{-} Z^{+}$, giving $E^{ \pm} \propto k^{-5 / 3}$ (in this shell model, which includes nonlinear interactions only locally in Fourier space). The resulting spectra change with distance, starting from a coherent-interaction-dominated spectrum at the coronal base and evolving toward the asymptotic
Kolmogorov spectra at greater distance, where reflection is negligible. According to this model, outside the Alfvénic critical point, the turbulent spectra have already lost any feature acquired in the low corona. Note that this is referred to perpendicular wavenumber spectra and not to the frequency spectra, which by contrast are almost unchanged, since their evolution is limited to the first solar radius above the coronal base.

Turbulent dissipation is remarkably high in the low corona. Depending on the injected energy an almost complete or partial matching is found with the "theoretical" heating, that is the one required to form the imposed background solar wind. The best agreement is found for $\delta u_{\odot} \approx 50 \mathrm{~km} \mathrm{~s}^{-1}$ which is at the limit of observational constraints (Chae et al. 1998). Nonetheless, even for more conservative values $\delta u_{\odot} \approx 40 \mathrm{~km} \mathrm{~s}^{-1}$, turbulent dissipation accounts for half of the above theoretical heating, maintaining the same profile (i.e., a peak at the sonic point). This implies that the role of coherent interactions is fundamental in shaping the heating function and that turbulence and turbulent heating cannot be neglected when studying the acceleration of the solar wind.

The peak dissipation seems to scale linearly with the rms amplitude $Z_{\odot}^{+}$although a proof of the precise scaling would require further studies. We finally observe that if the lower boundary is shifted to the base of the chromosphere, hence including the transition region, a stronger dissipation rate is expected to be found at the transition region and in the low corona. The Alfvén speed is smaller below the transition region but its gradients are higher, increasing the amount of energy residing in the anomalous reflected component which, having more time to interact with its mother wave, might increase the spectral energy transfer.

This research was supported in part by ASI contract n.I/ 015/07/0 "Solar System Exploration" and it was carried out in part at JPL under a contract from NASA. A. V. acknowledges support from the Belgian Federal Science Policy Office through the ESA-PRODEX program.

\section{REFERENCES}

Bavassano, B., Dobrowolny, M., Mariani, F., \& Ness, N. F. 1982, J. Geophys. Res., 87, 3616

Bavassano, B., Pietropaolo, E., \& Bruno, R. 2000a, J. Geophys. Res., 105, 12697 Bavassano, B., Pietropaolo, E., \& Bruno, R. 2000b, J. Geophys. Res., 105, 15959

Biskamp, D. 1994, Phys. Rev. E, 50, 2702

Buchlin, E., \& Velli, M. 2007, ApJ, 662, 701

Chae, J., Yun, H. S., \& Poland, A. I. 1998, ApJS, 114, 151

Cranmer, S. R., \& van Ballegooijen, A. A. 2005, ApJS, 156, 265

De Pontieu, B., et al. 2007, Science, 318, 1574

Dmitruk, P., Milano, L. J., \& Matthaeus, W. H. 2001, ApJ, 548, 482

Dmitruk, P., et al. 2002, ApJ, 575, 571

Grappin, R. 2002, J. Geophys. Res. (Space Phys.), 107, 1247

Grappin, R., Leorat, J., \& Pouquet, A. 1983, A\&A, 126, 51

Hansteen, V. H., Leer, E., \& Lie-Svendsen, Ø. 1999, in ESA SP-448, Magnetic Fields and Solar Processes, ed. A. Wilson et al. (Noordwijk: ESA), 1091

Heinemann, M., \& Olbert, S. 1980, J. Geophys. Res., 85, 1311

Hollweg, J. V. 1978, Sol. Phys., 56, 305

Hollweg, J. V., \& Isenberg, P. A. 2007, J. Geophys. Res. (Space Phys.), 112, 8102

Hollweg, J. V., Jackson, S., \& Galloway, D. 1982, Sol. Phys., 75, 35

Kopp, R. A., \& Holzer, T. E. 1976, Sol. Phys., 49, 43

Munro, R. H., \& Jackson, B. V. 1977, ApJ, 213, 874

Tomczyk, S., et al. 2007, Science, 317, 1192

Tu, C.-Y., Pu, Z.-Y., \& Wei, F.-S. 1984, J. Geophys. Res., 89, 9695

Van Doorsselaere, T., Nakariakov, V. M., \& Verwichte, E. 2008, ApJ, 676, L73 Velli, M. 1993, A\&A, 270, 304

Velli, M., Grappin, R., \& Mangeney, A. 1989, Phys. Rev. Lett., 63, 1807

Velli, M., Grappin, R., \& Mangeney, A. 1990, Comput. Phys. Commun., 59, 153

Verdini, A., \& Velli, M. 2007, ApJ, 662, 669 